\title{
Study on Micro Displacement Mechanism of Hydraulic Fracturing by Oil Displacement Agent at High Pressure
}

\author{
Fengjiao Wang $\mathbb{D}^{1,2}$ Xu Wang, ${ }^{1}$ Yikun Liu $\mathbb{D}^{1},{ }^{1}$ Qingjun Deng, ${ }^{3}$ Jianan $\mathrm{Hu}^{4}$ and Yingbo $\mathrm{Lv}^{4}$ \\ ${ }^{1}$ Laboratory of Enhanced Oil Recovery of Education Ministry, Northeast Petroleum University, Daqing, 163318 Heilongjiang, China \\ ${ }^{2}$ Daqing Oilfield Co. Ltd., Postdoctoral Res Ctr, Daqing Heilongjiang 163453, China \\ ${ }^{3}$ No. 1 Oil Production Plant CNPC (China), Daqing, Heilongjiang 163318, China \\ ${ }^{4}$ Fuyu Oil Production Plant, Jilin Oilfield, Songyuan, Jilin 138000, China
}

Correspondence should be addressed to Yikun Liu; liuyikun@nepu.edu.cn

Received 26 February 2021; Revised 27 May 2021; Accepted 5 July 2021; Published 23 July 2021

Academic Editor: Chuangzhou Wu

Copyright ( 2021 Fengjiao Wang et al. This is an open access article distributed under the Creative Commons Attribution License, which permits unrestricted use, distribution, and reproduction in any medium, provided the original work is properly cited.

\begin{abstract}
The type-III oil formations in Daqing Oilfield are the representatives of medium-low permeability reservoirs in ultrahigh water cut oilfields of China, which is characterized by bad connectivity of pores and throats, dispersed residual oil distribution, and difficult to displace effectively. In order to produce the residual oil, we propose a new EOR (enhanced oil recovery) method which is hydraulic fracturing by an oil displacement agent at high pressure. In this paper, firstly, we have performed three sets of displacement experiments under different conditions to provide the basis for the analysis of changes in core pore structure and wettability. Next, overburden pressure porosity and permeability tests were used to analyze the effect of the injection of an oil displacement agent at high pressure on core physical properties. Correspondingly, the constant speed mercury injection tests were used to determine the radius distribution of pore throat and change of seepage resistance under different displacement conditions. Moreover, the scanning electron microscopy (SEM) tests of cores were carried out to observe and analyze changes in porethroat size and connectivity, mineral particle accumulation, and cementation before and after hydraulic fracturing by an oil displacement agent at high pressure. Finally, core wettability tests were conducted to discuss and analyze the rule of core wettability change in hydraulic fracturing by an oil displacement agent at high pressure, and its mechanism of wettability changes. Research shows that increasing the formation energy is the most important mechanism of EOR by a fracturingseepage-displacement method. Additionally, the type of an oil displacement agent has less effect. After an oil displacement agent at high pressure is injected to fracture the formation, it not only provides efficient flow channel and larger sweep volume for an oil displacement agent. Under the flushing action of high-pressure injection fluid, the original way of line or point contact between mineral particles gradually changes to free particles. Therefore, the pore throat size increases, some larger pores are formed, and the overall flow resistance decreases. After the injection of fluid at high pressure, the energy in formation has increased and the core wettability changes from oil-wet to weakly water-wet. This is not only because the residual oil on the pore surface is flushed by high pressure; in addition, the adsorption of an oil displacement agent on the rock surface reduces the liquid-solid interface energy and changes the wettability, thus improving the oil displacement efficiency.
\end{abstract}

\section{Introduction}

Nowadays, about $70 \%$ of Chinese oil production is still exploited from the old oilfields. For a period of time in the future, the old oilfields will still be the main Chinese oil supply [1-5]. Take Daqing Oilfield of China as an example, at present, water driving is still the main way of oil develop- ment. The general water cut is about $92.7 \%$, but the recovery rate is only about $35 \%$ : thus, there is great potential for further EOR in this area [6-9]. Additionally, the remaining oil mainly exists in medium-low permeability reservoirs. While these reservoirs are always bad in physical properties and the distribution of remaining oil is relatively dispersed, it is difficult to inject the oil displacement agent and, definitely, 
the remaining oil is difficult to be produced $[10,11]$. Therefore, there is an urgent need for a reasonable oil formation improvement method that can effectively produce the oil and release the dispersed remaining oil.

The development experience of large oilfields in the United States and Russia shows that oil production is a process of gradual improvement, which is mainly reflected in the increasingly reservoir hierarchy division and the increasing density of well pattern [12-14]. With the continuous hierarchy division of reservoir and interwell infill in multilayer sandstone reservoir, the injection-production pressure system is constantly improved and strengthened. In the process of oil production, from primary oil recovery, secondary oil recovery to tertiary oil recovery, reservoir energy supplement has always been one of the most important methods to EOR [15-18]. Elastic energy recovery, dissolved gas driving, gas driving, steam huff and puff, and so on, the mechanism of EOR is essential to maintain or increase the formation energy. After all, enough pressure is the fundamental driving force of crude oil development; enough pressure difference ensures the successful exploitation of crude oil sustainable development.

Hydraulic fracturing, as a direct and effective stimulation measure, has been widely used in oilfields all over the world. With the exploration, development, and utilization of shales and other unconventional reservoirs, hydraulic fracturing has become a necessary means to result in complex fracture systems instead of simple planar fractures and provide flow channel for oil and gas [19-21]. For a long time, the fracturing fluid with high viscosity and low filtration has been widely used in the fracturing operation domestically and overseas [22-24]. These fracturing fluids generally have the advantages of high viscosity and low fluid loss. The commonly used fracturing fluids are represented by guanidine or modified guanidine. Vegetable gum water-based fracturing fluid is one of the most commonly used fracturing fluids, which is used earlier. Its high viscosity and low fluid loss can satisfy the needs of fracturing and carrying sand [25-27]. In order to further improve the fracturing performance effect, various fracturing fluid systems have been developed successively, such as crosslinked polymer gel fracturing fluid system [28], foam fracturing fluid system [29], VES fracturing fluid system $[30,31]$, cellulose fracturing fluid system, and so on [32]. These developments have improved the temperature resistance, shear resistance, sand carrying capacity, and wall building performance of the fracturing fluid system.

Since the first hydraulic fracturing well was constructed in 1947, hydraulic fracturing has always been used to establish a high-speed flow channel to increase oil and gas production and injection $[33,34]$. At the later stage of oilfield development, it is difficult to effectively use the dispersed remaining oil in medium-low permeability reservoirs. In view of the above problems, our research team proposed a new EOR method: hydraulic fracturing by an oil displacement agent with high pressure. In this method, the oil displacement agent with low initial viscosity is used as fracturing fluid, and the oil displacement agent is carried to the target reservoir by the way of hydraulic fracturing. The oil displacement agent is rapidly pushed to the enrichment posi- tion of remaining oil through fractures, so as to achieve the higher efficiency of oil displacement. The process of hydraulic fracturing is transformed into the process of fracturingseepage-oil displacement along the direction of perpendicular to the fracture. In this way, the displacement agent can quickly enter the pores; thus, the contact time and distance between the oil displacement agent and the formation can be shortened effectively. So it can solve the problems of higher fracturing fluid loss and lower utilization efficiency of the oil displacement agent in the traditional injection process. As we all know, the oil recovery depends on effective swept volume and oil displacement efficiency. Only when the swept volume reaches a certain extent, the oil displacement efficiency can be improved; only by effectively improving the liquid absorption capacity of medium-low permeability layers or small-medium pores in the reservoir can the swept volume be expanded and the oil recovery be greatly enhanced [3537]. This new EOR method of fracturing-seepage-oil displacement combines the advantages of increased formation pressure, expanded swept volume, and enhanced oil displacement efficiency.

In this paper, aiming at the hydraulic fracturing by an oil displacement agent with high pressure we proposed, a series of studies on micro displacement mechanism has been carried out. The natural core parameters, including permeability, porosity, pore throat structure, and wettability, were tested by overburden porosity and permeability instrument, constant speed mercury injection instrument, scanning electron microscope (SEM), contact angle instrument, and oil displacement device. We have designed a series of experiments to compare and analyze the effects on micropore structure of cores under different conditions, including oil displacement at conventional speed, oil displacement by water at high pressure, and oil displacement by oil agent displacement at high pressure. On this basis, we clarified the micro oil displacement mechanism of hydraulic fracturing by an oil displacement agent at high pressure.

\section{Materials and Methods}

2.1. Experimental Materials. In this EOR method, as the oil displacement agent was injected under the condition of high pressure and would fracture the reservoir, it could also be regarded as the fracturing fluid. The oil displacement agent was the surfactant (petroleum sulfonate), which was provided by the Daqing Oilfield Downhole Operation Branch Company. The fracturing fluid used in the comparison experiment was water. The water in the experiments was prepared in the on-site construction of the Downhole Operation Branch Company. The oil in the experiments was simulated oil, which was a mixture of degassed and dehydrated crude oil and light hydrocarbon oil in Daqing Oilfield. The viscosity of the simulated oil was $8.86 \mathrm{mPa} \cdot \mathrm{s}$ at $45^{\circ} \mathrm{C}$. The cores in the experiments were natural cores, which were taken from the type-III formations of No.1 oil production plant in Daqing Oilfield. The diameter of natural core was $2.5 \mathrm{~cm}$, and the permeability was in the range of $100 \times 10^{-3} \mu \mathrm{m}^{2}$ to $200 \times$ $10^{-3} \mu \mathrm{m}^{2}$. 
2.2. Instrument and Facilities. In this study, we carried out three sets of displacement experiments under different conditions to provide the basis for the analysis of changes in core pore structure and wettability at first. Based on them, overburden pressure porosity and permeability tests, core pore radius distribution test, microstructure change test, and wettability change test were performed. The main device used in the experiments included an overlaying pressure pore-permeability instrument, constant speed mercury injection instrument, scanning electron microscope (SEM), and contact angle tester. The microscopic pore structure parameters of the core were measured by a constant speed mercury injection instrument. A Fei Tecnai G2 F20 scanning electron microscope (SEM) from Gatan Company, USA, was used to test the micromorphology of natural cores. The equipment used in oil displacement experiments mainly includes an advection pump, pressure gauge, and oil displacement agent container. Except for the advection pump, the other facilities were placed in an oven with a constant temperature of $45^{\circ} \mathrm{C}$. The displacement pressure was provided by the advection pump, and the fluid in the intermediate container was injected into the cores. In order to compare the effects of conventional displacement and fracturing-seepage-displacement methods at high pressure on the microscopic pore throat structure and wettability changes of cores, we had designed three sets of experiments, including the following: (a) oil displacement at conventional speed, the injection rate was $0.1 \mathrm{~mL} / \mathrm{min}$ and the injection volume was $30 \mathrm{PV}$ (pore volume); (b) hydraulic fracturing by water flooding at high pressure, the injection pressure was $20 \mathrm{MPa}$ and the injection volume was $30 \mathrm{PV}$; (c) hydraulic fracturing by oil displacement agent flooding at high pressure, the injection pressure was $20 \mathrm{MPa}$ and the injection volume was $30 \mathrm{PV}$. Combined with the SEM test, the influence of different displacement conditions on the microstructure changes of cores was analyzed through the core samples after displacement obtained in this experiment. The schematic diagram of the experimental set-up is shown in Figure 1.

2.3. Experimental Methods. In this study, there were four experiments that were conducted to reveal the micro displacement mechanism of hydraulic fracturing by an oil displacement agent at high pressure to EOR. The detailed experimental methods and procedures of each experiment are as follows.

2.3.1. Methods for Measurement of Core Porosity and Permeability. The same natural core was cut into four segments of equal in length, and permeability and porosity tests were carried out under four different conditions. The experiment was performed with an overburden pressure pore-permeability instrument as shown in Figure 2. (1) A natural core with a diameter of about $2.5 \mathrm{~cm}$ was selected, and it should be dried; (2) the selected natural cores were cut into four parts with the same in length, and the target cores were loaded into the core gripper of the overburden pressure pore-permeability instrument; after setting the confining pressure parameters, the cores were loaded to measure the porosity; (3) the ratio of gas flow rate to core volume of the gas tank under constant pressure was calculated by computer, and the porosity could be obtained; (4) after the poros- ity measurement was over, the system began to enter the stage of the permeability measurement. The computer calculated the core permeability by calculating the pressure drop rate under the gas tank and other flow conditions.

\subsubsection{Conventional Mercury Injection Measurement of Core}

Parameters. The microscopic pore structure parameters of the core were measured by a constant speed mercury injection instrument as shown in Figure 3. (1) A natural core with a diameter of about $2.5 \mathrm{~cm}$ was selected, and it should be washed with toluene oil and then dried; (2) measurement of the physical properties of the target core, including permeability measured with gas, volume, mass, and density; (3) the target core was loaded into the constant speed mercury injection instrument, and mercury was injected under the set pressure. After the pressure stabilized, the pressure and mercury injection volume were recorded to improve the injection pressure, and the above experimental process was repeated. (4) The injection pressure was equal to the capillary pressure corresponding to the pore radius where mercury could be injected, and the capillary radius corresponding to the capillary pressure was equal to the pore throat radius of the core. By continuously increasing the injection pressure, the capillary pressure curve could be obtained, and the distribution probability of different pore radius could be calculated by combining the volume of mercury injected.

\subsubsection{Method for Measurement of Core Microscopic} Morphology. The core morphology was observed by a scanning electron microscope shown in Figure 4. (1) Core sample production: use a burette to absorb a small amount of core debris, evenly coated in clean and fixed sample box, serial reserve; (2) freezing and drying samples: the prepared samples were quickly transferred to the E7400cryotrans refrigerating platform, then slowly poured into liquid nitrogen for freezing and vacuuming, and then rapidly heated up. The water in the sample froze and sublimated to get dry samples; (3) gold spraying: the sample was placed in a certain vacuum high voltage electric field, the high voltage electric field ionizes the air, and then the sample surface was coated with a layer of conductive metal film; (4) electron microscope scanning: the samples were placed under the scanning electron microscope and observed in the sample room. Pictures were selected to observe the microscopic morphology of each sample, and the characteristics of different systems were evaluated and analyzed.

2.3.4. Core Wettability Analysis. The wettability experiment mainly used contact angle measuring instrument SDC-200S as shown in Figure 5. Wettability is the interaction between oil and water and reservoir rock under reservoir conditions, which determines the microscopic and original distribution state of reservoir fluid in rock channels and plays a determination role in the recovery of crude oil in oil production. (1) The columnar natural core was selected, and the core was ground until the surface was smooth. The titration volume of the microsyringe needle was set as $3.00 \mu \mathrm{L}$, the sample table was used to collect distilled water, and the camera was used to record this process; (2) after setting the baseline 


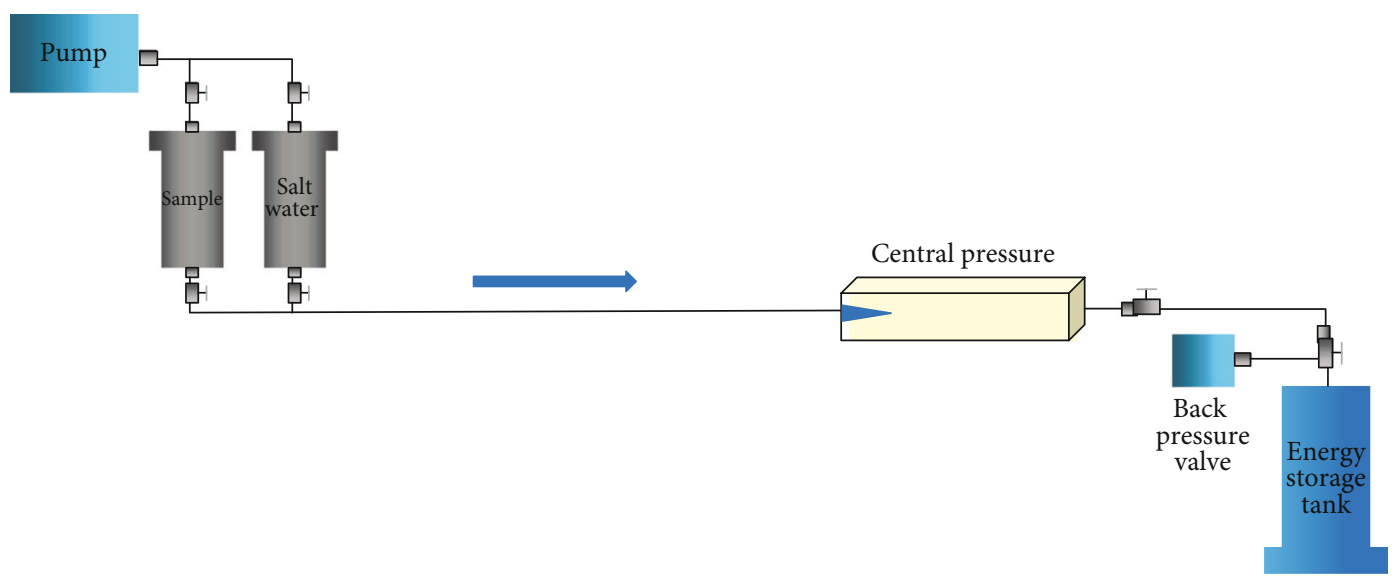

FIgURE 1: Schematic diagram of the experimental set-up.

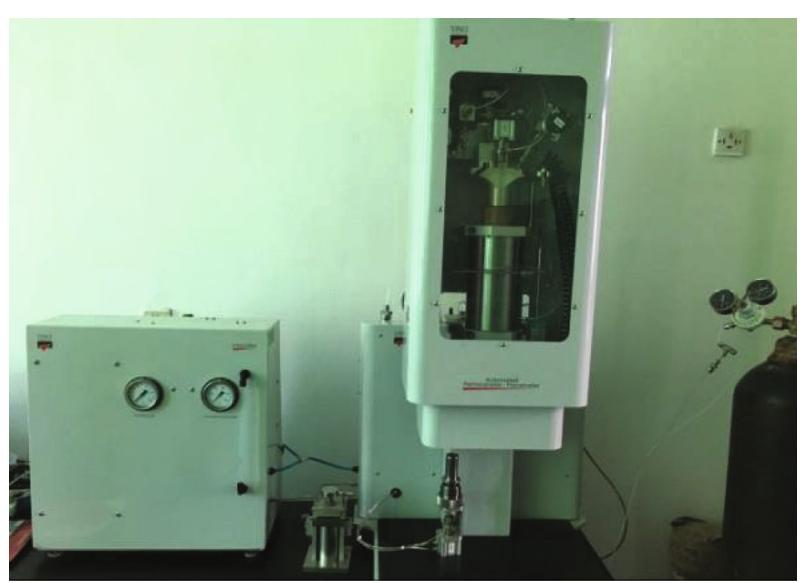

Figure 2: Overburden pressure porosity instrument.

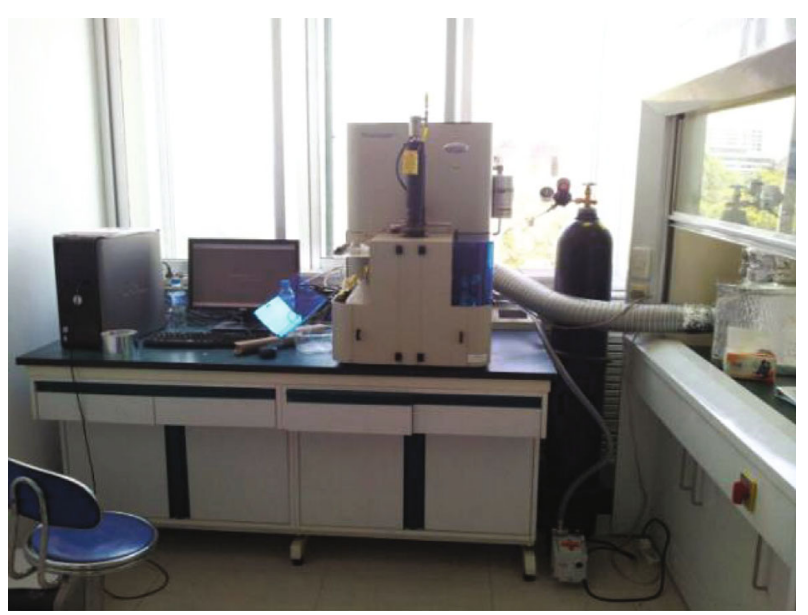

FIGURE 3: Constant speed mercury injection instrument.

position and the fitting function, the droplet contour could appear on the droplet image and the droplet connection could be obtained tentacles; (3) the systematic error of the effective result should be less than $5 \%$. In order to minimize the error, three different measuring points were randomly selected on the same core, and the average value of the effec- tive result was taken as the core contact angle; (4) the core was taken out after soaking in distilled water for a period of time, and the change of foundation angle after soaking was measured after drying. (5) The system error of the contact angle measuring instrument was less than 5\%; (6) measurement of wettability after conventional water flooding: the wettability of the core after displacement was measured, i.e., steps (1)-(5) were repeated. (7) Measurement of core wettability after fracturing by water flooding formed at high pressure: steps (1)-(5) were repeated. (8) Measurement of core wettability after fracturing by surfactant flooding formed at high pressure: steps (1)-(5) were repeated.

The self-priming method was used to measure the core wettability index as shown in

$$
\begin{gathered}
I_{\mathrm{w}}=\frac{w_{\mathrm{a}}}{w_{\mathrm{a}}+w_{\mathrm{w}}}, \\
I_{\mathrm{o}}=\frac{w_{\mathrm{o}}}{w_{\mathrm{o}}+w_{\mathrm{b}}}, \\
I_{\mathrm{w}-\mathrm{o}}=I_{\mathrm{w}}-I_{\mathrm{o}},
\end{gathered}
$$

where $I_{\mathrm{w}}$ denotes the wettability index of oil phase; $I_{\mathrm{o}}$ denotes the wettability index of water phase; $I_{\mathrm{w}-\mathrm{o}}$ denotes the AmottHarvey index; $w_{\mathrm{a}}$ denotes the amount of crude oil discharged by spontaneous absorption; $w_{\mathrm{w}}$ denotes the amount crude oil discharged by water driving; $w_{\mathrm{o}}$ denotes the amount of water discharged by spontaneous oil absorption; $w_{\mathrm{b}}$ denotes the amount of water discharged by oil flooding.

The wettability grading standards are shown in Table 1.

\section{Results and Discussions}

3.1. Test Results of Porosity and Permeability Changes in Natural Cores. The natural cores 17-1 and 21-2 of the typeIII formations of the No.1 oil production plant in Daqing Oilfield were selected and quartered. They tested the porosity and permeability of the cores in its original state, conventional water injection displacement, hydraulic fracturing by water flooding at high pressure, and hydraulic fracturing by oil displacement agent flooding at high pressure. The core parameter test results are shown in Table 2. 


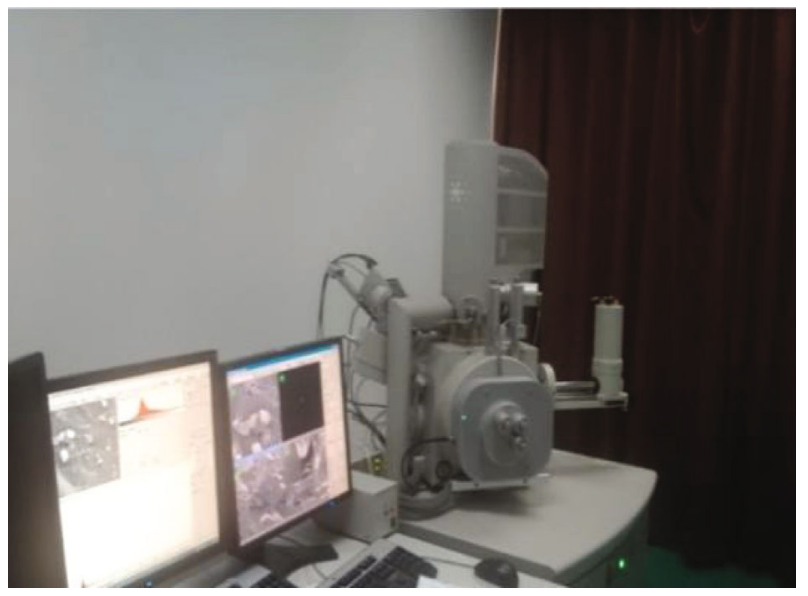

FIgURE 4: FEI Tecnai G2 20 scanning electron microscope.

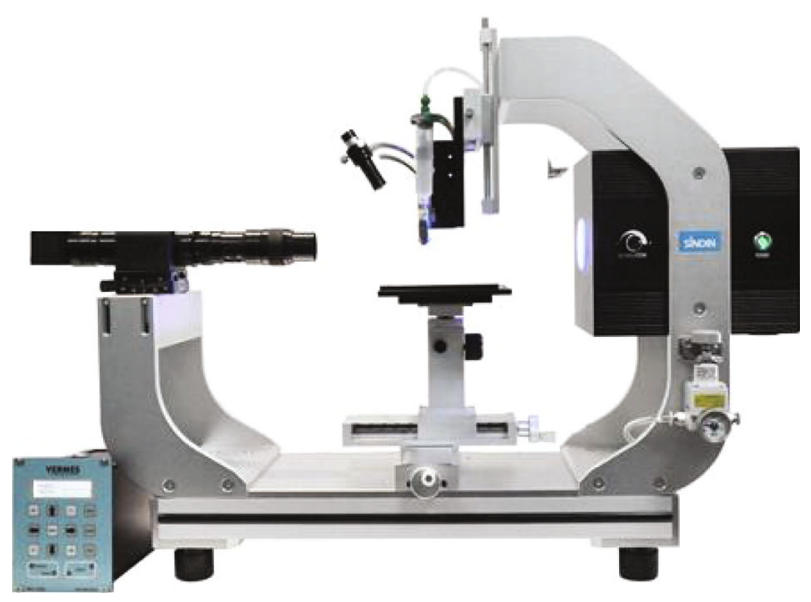

FIGURE 5: SDC-200S contact angle tester.

The measurement results of porosity and permeability under different displacement conditions of natural cores 17-1 and 21-2 are as shown in Figures 6 and 7, respectively. Obviously, for the cores in the original state and after water flooding under conventional injection rate, the porosity and permeability changed little. However, for the cores under hydraulic fracturing by water or an oil displacement agent at high pressure, the porosity and permeability were significantly increased. In particular, the permeability of core 17-1 and core 21-2 was increased by about $50 \times 10^{-3} \mu \mathrm{m}^{2}$ after displacement at high pressure. It showed that high injection pressure greatly improved the percolation capacity of the reservoirs. It is worth noting that the change of core porosity and permeability parameters was mainly related to the increase of reservoir energy and high-pressure flushing, but less affected by the injected fluid. Namely, whether the injection fluid was water or oil displacement agent, the effect was not obvious.

3.2. Test Results of Core Pore Structure by SEM. The pore structure tests of natural cores were performed by SEM under four states, including original state, conventional water injection displacement (injection rate: $0.1 \mathrm{~mL} / \mathrm{min}, 30 \mathrm{PV}$ ), hydraulic fracturing by water flooding at high pressure (injection pressure: $20 \mathrm{MPa}, 30 \mathrm{PV}$ ), and hydraulic fracturing by an oil displacement agent at high pressure (injection pressure: $20 \mathrm{MPa}, 30 \mathrm{PV}$ ). The experimental results of core 17-1 are shown in Figure 8.

Figure 8(a) shows the original state of the type-III core before fracturing. The pores were filled with kaolinite, and the clay on the surface of the particles was not obvious. Additionally, the development phenomenon of intergranular pores was not obvious and the mineral particles contacted with each other in the form of points or lines. After conventional injection water displacement as shown in Figure 8(b), the occurrence state and pore form of the mineral had no obvious change. The main components of the core were complete, and no feldspar was damaged or corroded. However, when the core was displaced by high-pressure injection fluid as shown in Figures 8(c) and 8(d), the supporting mode of the skeleton particles had changed. The cementing materials at the cementation between particles were migrated to other parts under the high-pressure injection fluid washing. The original contact relationship between the particles gradually changed to the contact mode with free particles, and the number of connected pores and throat increased. The experimental results showed that in the process of fracturing and oil displacement, the fracturing fluid stored energy at the fracture after hydraulic fracturing, and the fracturing fluid formed a large energy field at the fracture and percolated into the matrix. In the process of fracturing-seepage-displacement, the permeability and porosity of natural core increased. The change of core basic parameters was mainly related to energy enhancement.

3.3. Test Results of Conventional Mercury Injection. In order to study the change of pore structure of matrix cores under different displacement methods, a conventional mercury injection instrument was used to measure the natural cores of type-III formations of Daqing Oilfield. The mercury injection test results of the core under the original state are shown in Figure 9. The test results of hydraulic fracturing by oil displacement agent (surfactant) flooding at high pressure are shown in Figure 10. Table 3 shows the measurement results of core pore result parameters under different displacement conditions.

The results showed that the maximum pore radius and average pore radius of the cores became larger, and the pore distribution range became smaller, and the pore distribution tended to be more stable as the fracturing fluid flowed into the matrix under the action of increasing energy of the fracturing fluid. After injecting the oil displacement agent at high pressure, the greater the contribution rate of pore and throat to permeability in the range of $6.3 \mu \mathrm{m}$ to $10 \mu \mathrm{m}$, the higher the distribution frequency of pore throat radius in this range. At present, the main outlet channel radius and effective seepage channel radius were usually used to describe the contribution of pore throat radius to permeability. The outlet channel radius referred to the distribution range of pore throat radius corresponding to the peak value of permeability contribution distribution curve. Before oil displacement agent flooding at high pressure, the distribution frequency 
TABLE 1: The wettability grading standards.

\begin{tabular}{|c|c|c|c|c|c|}
\hline \multirow{2}{*}{ Wettability index } & \multicolumn{5}{|c|}{ Wettability grade } \\
\hline & Oil-wet & Weak oil-wet & Neutral & Weak water-wet & Water-wet \\
\hline The wettability index of oil phase & $1 \sim 0.8$ & $0.7 \sim 0.6$ & \multirow{2}{*}{ Approximate } & $0.3 \sim 0.4$ & $0 \sim 0.2$ \\
\hline The wettability index of water phase & $0 \sim 0.2$ & $0.3 \sim 0.4$ & & $0.7 \sim 0.6$ & $1 \sim 0.8$ \\
\hline$I=I_{\mathrm{w}}-I_{\mathrm{o}}$ & & $0.1]$ & $(-0.1,0.1)$ & & \\
\hline
\end{tabular}

TABLE 2: Results of porosity and permeability measurements in natural cores.

\begin{tabular}{|c|c|c|c|c|}
\hline $\begin{array}{l}\text { Core } \\
\text { number }\end{array}$ & The experimental scheme & $\begin{array}{c}\text { Porosity measured with } \\
\text { gas (\%) }\end{array}$ & $\begin{array}{l}\text { Overburden pressure } \\
\text { porosity }(\%)\end{array}$ & $\begin{array}{l}\text { Effective permeability } \\
\qquad\left(\times 10^{-3} \mu \mathrm{m}^{2}\right)\end{array}$ \\
\hline \multirow{4}{*}{$17-1$} & Original state (predisplacement) & 23.8 & 22.4 & 86.0 \\
\hline & $\begin{array}{l}\text { Water driving under conventional injection } \\
\text { rate }\end{array}$ & 24.0 & 22.7 & 93.2 \\
\hline & Hydraulic fracturing by water at high pressure & 25.5 & 24.5 & 134.6 \\
\hline & $\begin{array}{l}\text { Hydraulic fracturing by oil displacement agent } \\
\text { at high pressure }\end{array}$ & 25.0 & 24.2 & 127.4 \\
\hline \multirow{4}{*}{$21-2$} & Original state (predisplacement) & 24.6 & 23.8 & 113.6 \\
\hline & $\begin{array}{l}\text { Water driving under conventional injection } \\
\text { rate }\end{array}$ & 25.0 & 24.1 & 120.2 \\
\hline & Hydraulic fracturing by water at high pressure & 28.2 & 26.3 & 166.3 \\
\hline & $\begin{array}{l}\text { Hydraulic fracturing by oil displacement agent } \\
\text { at high pressure }\end{array}$ & 29.4 & 26.5 & 175.5 \\
\hline
\end{tabular}
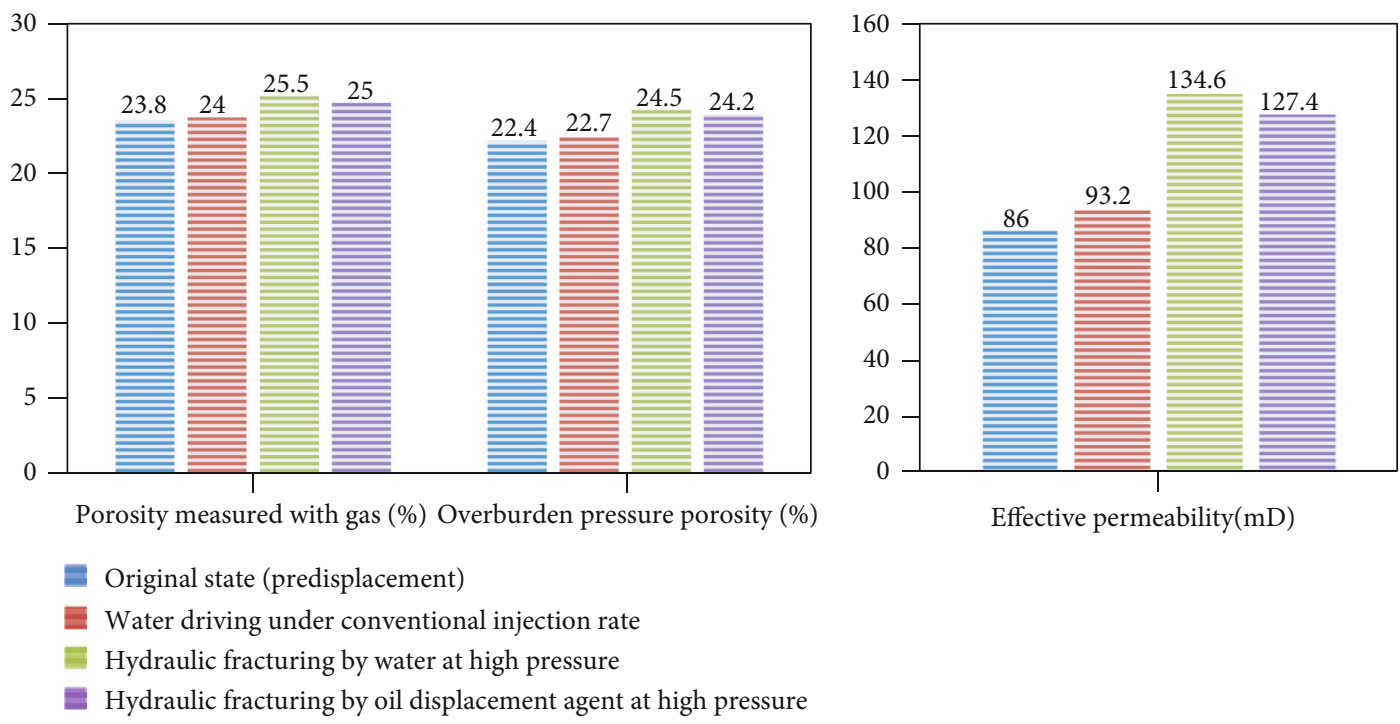

FIGURE 6: Results of porosity and permeability measurements of core 17-1.

peak of pore throat radius appeared in the range of $4 \mu \mathrm{m}$ to $6.3 \mu \mathrm{m}$; after oil displacement agent flooding at high pressure, the distribution frequency peak of pore throat radius appeared in the range of $6.3 \mu \mathrm{m}$ to $10 \mu \mathrm{m}$. This showed that highpressure displacement made the core pore size generally increase, the fluid flow resistance in the core became smaller, and the seepage capacity was improved.

3.4. Test and Analysis Results of Core Wettability Test. In order to study the influence of the high-pressure oil displace- ment agent on the wettability of matrix core, contact angle tests under different displacement conditions were carried out. Contact angle test results of core 17-1 are shown in Figure 11. According to Equations (1)-(3), we calculated the wetting index under four experimental conditions of core 17-1 and core 21-2, as shown in Figure 12.

The natural cores of the type-III formation in the No.1 oil production plant of Daqing Oilfield were of weak oil-wet type as shown in Figure 11(a), and the wettability of the cores was still of oil-wet type after water flooding under conventional 


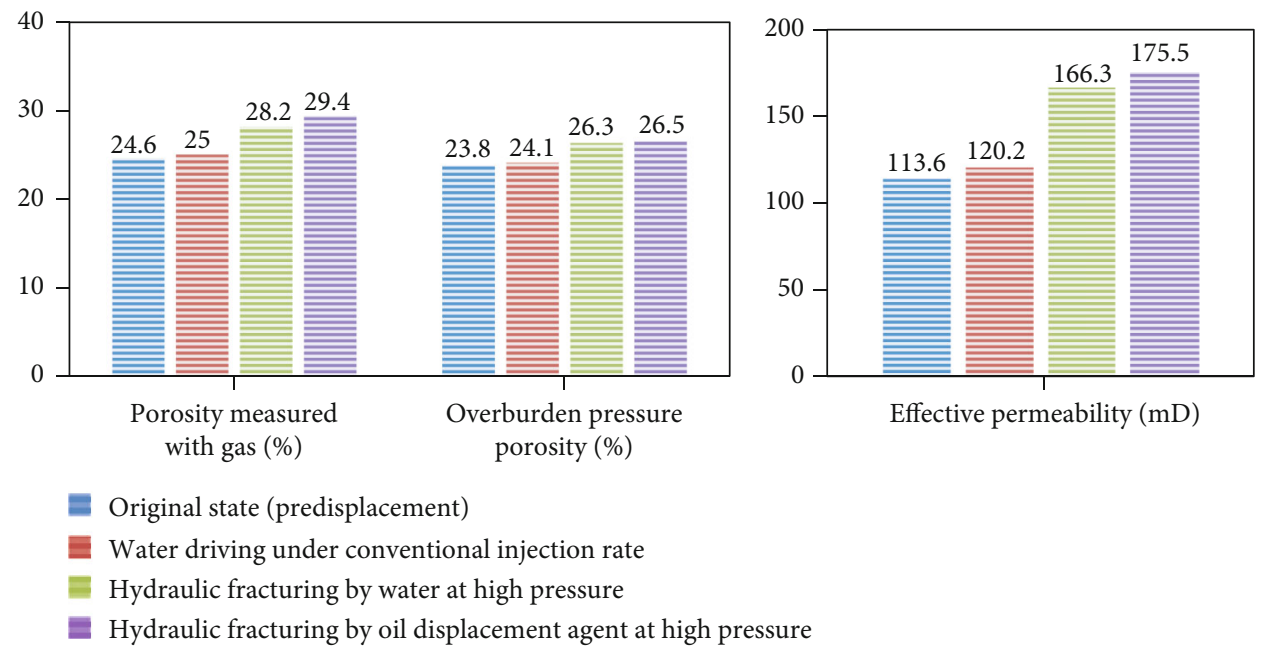

FIGURE 7: Results of porosity and permeability measurements of core 21-2.

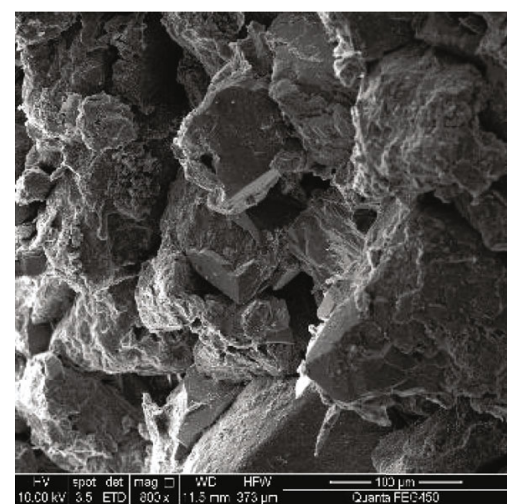

(a) Original state (before fracturing)

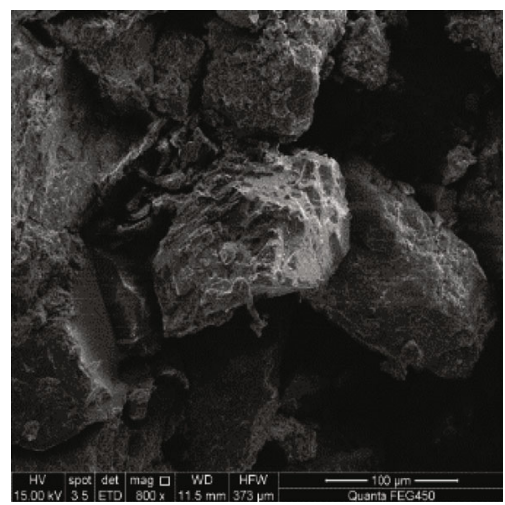

(c) Fracturing by water at high pressure

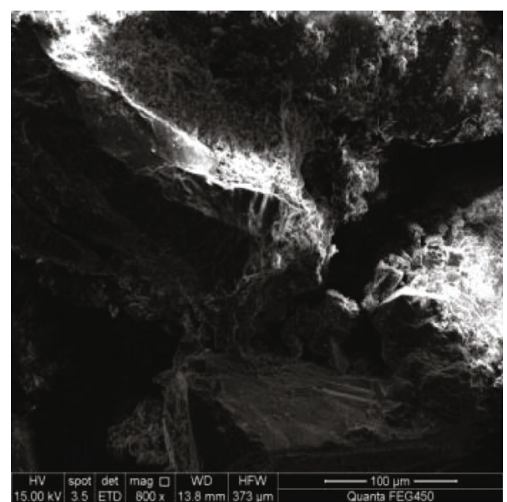

(b) Conventional water injection displacement

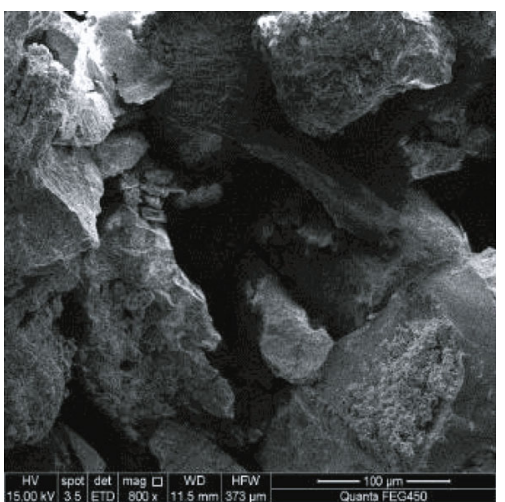

(d) Fracturing by an oil displacement agent at high pressure

Figure 8: Changes of core pore structure in different states of core 17-1.

injection rate as shown in Figure 11(b). After injecting water into the core at high pressure, the wetting angle decreased obviously, and the wettability of pore wall changed from oil-wet to weak water-wet as shown in Figure 11(c). In addition, when the injected fluid at high pressure was replaced by an oil displacement agent (surfactant in this paper), the wet- ting angle of the core wall decreased further as shown in Figure 11(d). It was the combined effect of high-pressure injection of an oil displacement agent and the surfactant on reducing interfacial tension. From the change of wettability index of core 17-1 and core 21-2, the average value also changed from negative to positive after high-pressure injection. 

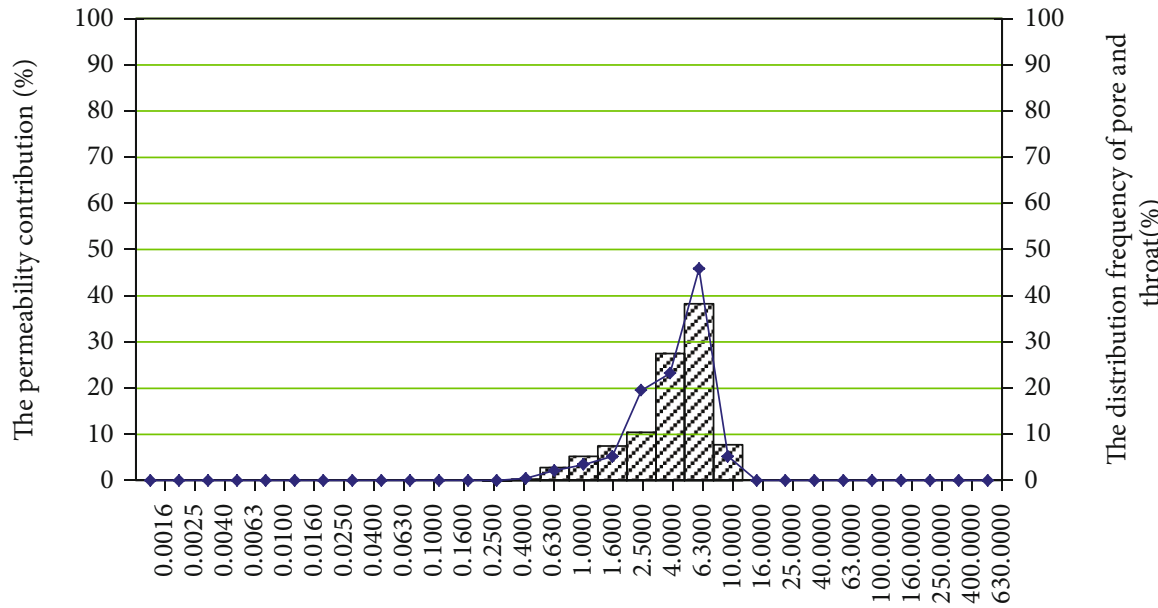

Distribution range of pore and throat $(\mu \mathrm{m})$

एकe distribution frequency of pore and throat (\%)

$\rightarrow$ The permeability contribution (\%)

Figure 9: Core pore radius distribution results under the original state of core 21-2.
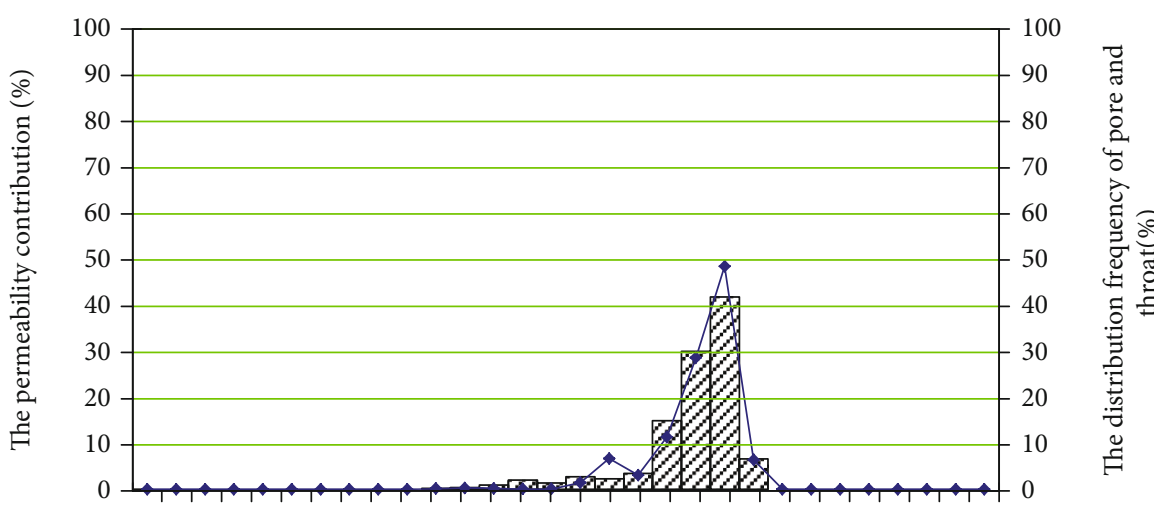

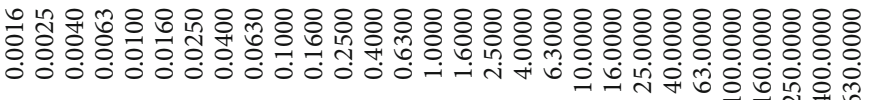

The distribution range of pore and throat $(\mu \mathrm{m})$

एक्य The distribution frequency of pore and throat (\%)

The permeability contribution (\%)

FIGURE 10: Core pore radius distribution results under the state of hydraulic fracturing by an oil displacement agent (surfactant) with high flow rate displacement of core 21-2.

TABLE 3: The measurement results of core pore parameters under different displacement methods.

\begin{tabular}{lcc}
\hline Experimental scheme & Maximum pore radius $(\mu \mathrm{m})$ & Average pore radius $(\mu \mathrm{m})$ \\
\hline Core original parameters (before displacement) & 13.82 & 4.736 \\
Core original parameters (after fracturing and driving by surfactant) $(20 \mathrm{MPa})$ & 21.23 & 7.401 \\
\hline
\end{tabular}




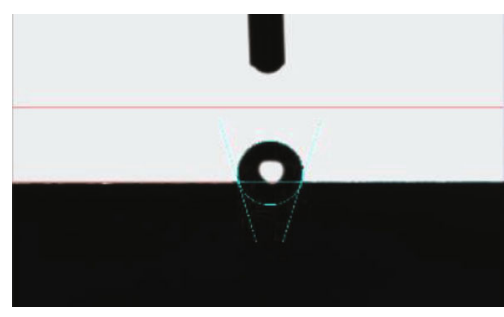

(a) Contact angle $=102.6^{\circ}$

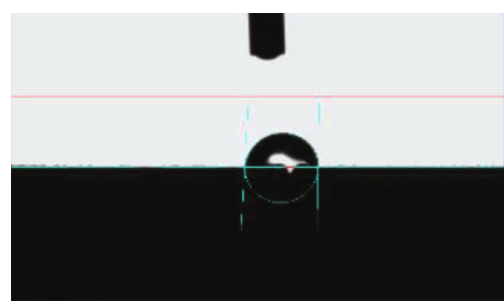

(c) Contact angle $=87.5^{\circ}$

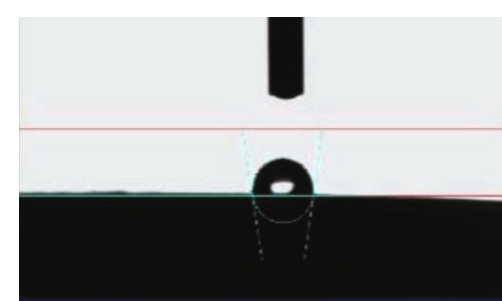

(b) Contact angle $=98.7^{\circ}$

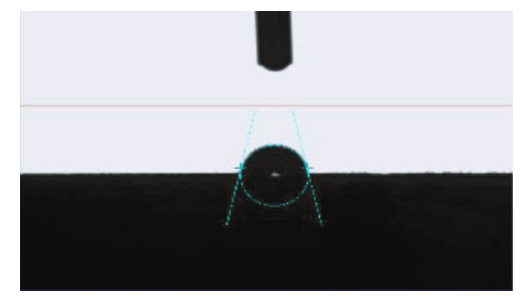

(d) Contact angle $=80.6^{\circ}$

FIgURE 11: The measurement of contact angle test of core 17-1.

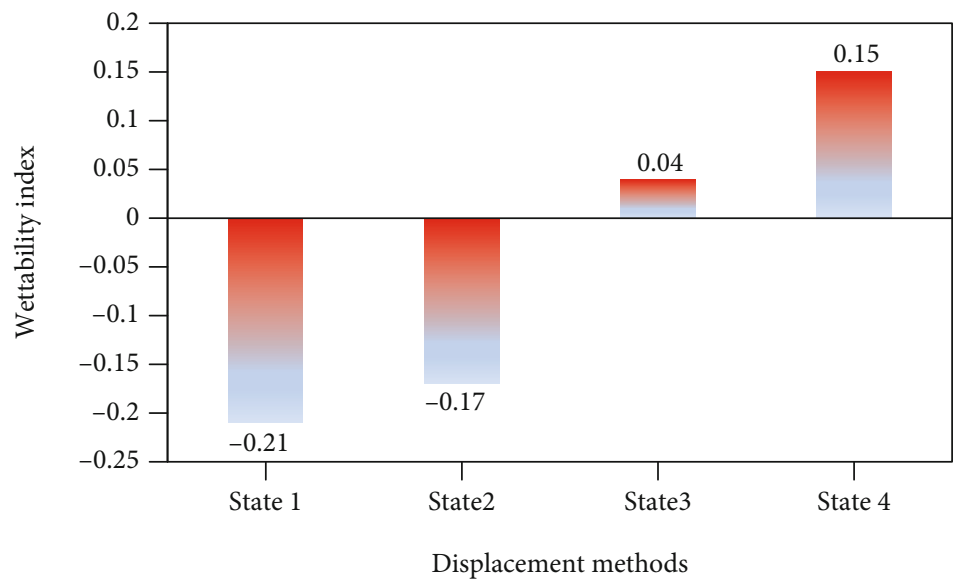

FIgURE 12: Average wetting index of core 17-1 and core 21-2 under four states (state 1 denotes the original state; state 2 denotes the state of water driving under conventional injection rate; state 3 denotes the state of fracturing by water at high pressure; state 4 denotes the state of fracturing by an oil displacement agent at high pressure).

On the one hand, injection fluid at high pressure effectively increased the formation energy, and the residual oil on the pore wall was stripped due to high-speed injection which was the main EOR mechanism of this method; on the other hand, the adsorption area of surfactant molecules on the pore surface was increasing, and the adsorption was dominated by monolayer, which reduced the liquid-solid interface energy and caused the wetting inversion of pore surface, thus improving the oil displacement efficiency. Besides, the type of injection fluid had little effect on it.

In a word, from the perspective of EOR, it may be the best choice to improve sweep volume and oil displacement efficiency at the same time. However, from the point of view of practical application in oilfields, the proposed approach is water injection at high pressure in general. The hydraulic fracturing method is used to bring the displacement fluid to the formation and provide energy to it.

\section{Conclusions}

(1) The most important mechanism of EOR by the fracturing-seepage-displacement method is to increase the formation energy by high-pressure injection of an oil displacement agent. Injection fluid displacement at high pressure can significantly increase the core permeability and porosity. It is worth mentioning that the type of injection fluid has little effect

(2) In the process of fracturing and oil displacement, the injection fluid forms a large energy field around the 
fracture and then, it will penetrate into the matrix at high-pressure injection of an oil displacement agent. The original way of line or point contact between mineral particles gradually changes to free particles. Therefore, the pore throat size increases, some larger pores are formed, and the overall flow resistance decreases

(3) After injection of an oil displacement agent at high pressure, the phenomenon of wetting inversion occurs at the pore wall, which changes from oil-wet to weakly water-wet. The main reason is the highpressure scouring of injected fluid, which leads to the stripping of residual oil on the pore wall. Besides, if the injected fluid is surfactant, it will also play a role in reducing the interfacial tension. In this way, the displacement efficiency has been improved

\section{Data Availability}

The data used to support the findings of this study are included within the article.

\section{Conflicts of Interest}

The authors declare that they have no conflicts of interest regarding the publication of this paper.

\section{Acknowledgments}

This research was supported by the National Natural Science Foundation of China (Youth Project) (Grant No. 51804076), China Postdoctoral Science Foundation Postdoctoral (No. 2021M690528), Projects in Heilongjiang Province (Grant No. LBH-Z20035), Youth Science Foundation of Northeast Petroleum University (Grant No. 2019NL-02), and Research Initiation Foundation of Northeast Petroleum University (Grant No. 2019KQ15).

\section{References}

[1] Q. L. Du, "Variation law and microscopic mechanism of permeability in sandstone reservoir during long-term water flooding development," Acta Petrolei Sinica, vol. 37, no. 9, pp. 1151164, 2016.

[2] S. Yuan and Q. Wang, "New progress and prospect of oilfields development technologies in China," Petroleum Exploration and Development, vol. 45, no. 4, pp. 698-711, 2018.

[3] L. Sun, X. Wu, W. Zhou, X. Li, and P. Han, "Technologies of enhancing oil recovery by chemical flooding in Daqing oilfield, NE China," Petroleum Exploration and Development, vol. 45, no. 4, pp. 673-684, 2018.

[4] D. Jia, H. Liu, J. Zhang et al., "Data-driven optimization for fine water injection in a mature oil field," Petroleum Exploration and Development, vol. 47, no. 3, pp. 674-682, 2020.

[5] C. Jiaqing, W. Qiangqiang, X. Jianhong et al., "Development status and prospect of water pre-separation technology for produced liquid in high water-cut oil well," Acta Petrolei Sinica, vol. 41, no. 11, pp. 1434-1444, 2020.

[6] X. Gao, S. O. Xinmin, M. Xinli et al., "Quantitative characterization of the hidden remaining oil controlled by reservoir architecture at extra high water cut stage," Acta Petrolei Sinica, vol. 37, no. z2, pp. 99-111, 2016.

[7] J. Wang, C. Shi, S. Ji, G. Li, and Y. Chen, "New water drive characteristic curves at ultra-high water cut stage," Petroleum Exploration and Development, vol. 44, no. 6, pp. 1010-1015, 2017.

[8] X. Wang, H. Dang, and T. Gao, "Method of moderate water injection and its application in ultra-low permeability oil reservoirs of Yanchang oilfield, NW China," Petroleum Exploration and Development, vol. 45, no. 6, pp. 1094-1102, 2018.

[9] Q. N. Yu, Y. K. Liu, S. Liang, S. Tan, Z. Sun, and Y. Yu, "Mechanism experiment of enhanced oil recovery by surfactant flooding: a case study of Daqing placanticline oilfield," Petroleum Exploration and Development, vol. 46, no. 6, pp. 1138 1147, 2019.

[10] M. Zhou, J. Bu, J. Wang, X. Guo, J. Huang, and M. Huang, "Study on three phase foam for enhanced oil recovery in extra-low permeability reservoirs," Oil \& Gas Science and Technology, vol. 73, no. 8, p. 55, 2018.

[11] Y. Long, R. Wang, B. Zhu et al., "Enhanced oil recovery by a suspension of core-shell polymeric nanoparticles in heterogeneous low-permeability oil reservoirs," Nanomaterials, vol. 9, no. 4, p. 600, 2019.

[12] Y. S. Gurav, A. Y. Dandekar, S. L. Patil, and S. D. Patwardhan, "Application of design of experiments for well pattern optimization in Umiat oil field: a natural petroleum reserve of Alaska case study," in SPE/IATMI Asia Pacific Oil \& Gas Conference and Exhibition, Bali, Indonesia, 2019.

[13] D. A. Novikov, S. A. Pavlova, D. Yu Kuznetsov, S. V. Ryzhkova, and A. A. Chernikov, "Contemporary development state of reservoir Yu1, the Verkh-Tarskoe oilfield (south of Western Siberia)," Journal of Physics: Conference Series, vol. 1451, no. 1, article 012008, 2020.

[14] J. Bingyu, "Progress and prospects of enhanced oil recovery technologies at home and abroad," Oil \& Gas Geology, vol. 33, no. 1, pp. 111-117, 2012.

[15] J. Lu, P. J. Liyanage, S. Solairaj et al., "New surfactant developments for chemical enhanced oil recovery," Journal of Petroleum Science \& Engineering, vol. 120, pp. 94-101, 2014.

[16] E. Georges Lwisa and A. R. Abdulkhalek, "Enhanced oil recovery by nitrogen and carbon dioxide injection followed by low salinity water flooding for tight carbonate reservoir: experimental approach," IOP Conference Series: Materials Science and Engineering, vol. 323, p. 012009, 2018.

[17] Q. You, Q. Wen, J. Fang, M. Guo, Q. Zhang, and C. Dai, "Experimental study on lateral flooding for enhanced oil recovery in bottom- water reservoir with high water cut," Journal of Petroleum Science and Engineering, vol. 174, pp. 747756, 2019.

[18] O. Massarweh and A. S. Abushaikha, "The use of surfactants in enhanced oil recovery: a review of recent advances," Energy Reports, vol. 6, pp. 3150-3178, 2020.

[19] F. Zhang, B. Damjanac, and S. Maxwell, "Investigating hydraulic fracturing complexity in naturally fractured rock masses using fully coupled multiscale numerical modeling," Rock Mechanics and Rock Engineering, vol. 52, no. 12, pp. 51375160, 2019.

[20] F. Zhang, E. Dontsov, and M. Mack, "Fully coupled simulation of a hydraulic fracture interacting with natural fractures with a hybrid discrete-continuum method," International Journal 
Numerical and Analytical Methods in Geomechanics, vol. 41, no. 13, pp. 1430-1452, 2017.

[21] F. Zhang, X. Wang, M. Tang et al., "Numerical investigation on hydraulic fracturing of extreme limited entry perforating in plug-and-perforation completion of shale oil reservoir in Changqing oilfield, China," Rock Mechanics and Rock Engineering, vol. 54, no. 6, pp. 2925-2941, 2021.

[22] T. Guo, S. Zhang, Z. Qu, T. Zhou, Y. Xiao, and J. Gao, "Experimental study of hydraulic fracturing for shale by stimulated reservoir volume," Fuel, vol. 128, pp. 373-380, 2014.

[23] R. Barati and J.-T. Liang, "A review of fracturing fluid systems used for hydraulic fracturing of oil and gas wells," Journal of Applied Polymer Science, vol. 131, no. 16, pp. 318-323, 2014.

[24] Y. Guo, B. Fang, Y. Luo, X. Qiu, B. Guan, and K. Li, "Research progress of high temperature fracturing fluid," Oilfield Chemistry, vol. 35, no. 3, pp. 545-549, 2018.

[25] H. Liu and D. Xiao, "A novel low-damage vegetable gumbased fracturing fluid and its application in low-permeability reservoirs," Acta Petrolei Sinica, vol. 33, no. 3, pp. 343-350, 2008.

[26] M. Alohaly, A. BinGhanim, R. Rahal, and S. Rahim, "Seawater fracturing fluid development challenges: a comparison between seawater-based and freshwater-based fracturing fluids using two types of guar gum polymers," in Spe Kingdom of Saudi Arabia Technical Symposium \& Exhibition, Dammam, Saudi Arabia, 2016.

[27] C. Wang, Z. Zhang, J. du, X. Li, M. Zhao, and Z. Zhang, “Titanium-based nanoscale cross-linker for guar gum fracturing fluid: effects on rheological behaviour and proppant-carrying ability," Micro \& Nano Letters, vol. 14, no. 10, pp. 10961101, 2019.

[28] M. Zhao, Y. Li, Z. Xu et al., "Dynamic cross-linking mechanism of acid gel fracturing fluid," Colloids and Surfaces A Physicochemical and Engineering Aspects, vol. 607, p. 125471, 2020.

[29] K. Zhang, Z. Zhao, M. Tang et al., "A new type of experimentally proposed in situ heat/gas clean foam fracturing fluid system," Journal of Petroleum Exploration and Production Technology, vol. 10, no. 8, pp. 3419-3436, 2020.

[30] Y. Chen, T. Pope, and J. Lee, "Novel $\mathrm{CO}_{2}$-emulsified viscoelastic surfactant fracturing fluid system," in SPE European Formation Damage Conference, Sheveningen, The Netherlands, 2005.

[31] W. Kang, S. J. Mushi, H. Yang, P. Wang, and X. Hou, "Development of smart viscoelastic surfactants and its applications in fracturing fluid: a review," Journal of Petroleum Science and Engineering, vol. 190, article 107107, 2020.

[32] Z. Gao, C. Dai, X. Sun, Y. Huang, M. Gao, and M. Zhao, "Investigation of cellulose nanofiber enhanced viscoelastic fracturing fluid system: increasing viscoelasticity and reducing filtration," Colloids and Surfaces A: Physicochemical and Engineering Aspects, vol. 582, pp. 123938-123938, 2019.

[33] J. D. Bredehoeft, R. G. Wolff, W. S. Keys, and E. Shuter, "Hydraulic fracturing to determine the regional in situ stress field, Piceance Basin, Colorado," Geological Society of America Bulletin, vol. 87, no. 2, p. 250, 1976.

[34] T. W. Teklu, X. Li, Z. Zhou, N. Alharthy, L. Wang, and H. Abass, "Low-salinity water and surfactants for hydraulic fracturing and EOR of shales," Journal of Petroleum Science \& Engineering, vol. 162, pp. 367-377, 2018.

[35] S. Pingping, Y. Shiyi, D. Baorong, S. Jie, and S. Kuiyou, "Influence factors of oil displacement efficiency and sweep effei- ciency in chemical flooding," Petroleum Exploration and Development, vol. 31, no. 1, pp. 1-4, 2004.

[36] J. Geng, H. Fan, Y. Zhao, and W. Kang, “A correlation between interfacial tension, emulsifying ability and oil displacement efficiency of ASP system for Daqing crude oil," Petroleum Science \& Technology, vol. 36, no. 24, pp. 2151-2156, 2018.

[37] Z. Liu, Y. Li, R. Leng, Z. Liu, X. Chen, and H. Hejazi, "Effects of pore structure on surfactant/polymer flooding-based enhanced oil recovery in conglomerate reservoirs," Petroleum Exploration and Development, vol. 47, no. 1, pp. 134-145, 2020. 